\title{
Nebula around R Corona Borealis
}

\author{
N. Kameswara Rao ${ }^{1,2}$ and David L. Lambert ${ }^{1}$ \\ ${ }^{1}$ The W. J. McDonald Observatory,The University of Texas, Austin, USA \\ ${ }^{2}$ Instituto de Astrofsica de Canarias, IAC. La Laguna, Tenerife, Spain
}

\begin{abstract}
The star R Corona Borealis (R CrB) shows forbidden lines of [O II], [N II], and [S II] during the deep minimum when the star is fainter by about 8 to 9 magnitudes from normal brightness, suggesting the presence of nebular material around it. We present low and high spectral resolution observations of these lines during the ongoing deep minimum of $\mathrm{R} \mathrm{CrB}$, which started in July 2007. These emission lines show double peaks with a separation of about $170 \mathrm{~km} / \mathrm{s}$. The line ratios of [S II] and [O II] suggest an electron density of about $100 \mathrm{~cm}^{-3}$. We discuss the physical conditions and possible origins of this low density gas. These forbidden lines have also been seen in other R Coronae Borealis stars during their deep light minima and this is a general characteristic of these stars, which might have some relevance to their origins.
\end{abstract}

Keywords. stars: general

\section{Introduction}

The $\mathrm{R}$ Coronae Borealis (RCB hereafter) stars are notable for two distinct characteristics: (1) they are hydrogen-poor (by a factor ranging from $\sim 10$ to $10^{8}$ ), helium-rich, carbon-rich, F-G(K) supergiants; (2) they undergo large declines in light (2 to 8 magnitudes) at irregular intervals due to formation of clouds of carbon dust in the line of sight to the star.

During the deep light minimum of RCBs, the star is almost completely obscured (by dust) and the environment of the star is revealed. The spectrum at minimum light shows a set of broad emission lines, among other things, (FWHM 150-200 km/s) that emerge when the star becomes faint (5 magnitudes or more), consisting of He I, Ca II, Na I D lines, and forbidden lines of $[\mathrm{N} \mathrm{II}],[\mathrm{O} \mathrm{II}],[\mathrm{S} \mathrm{II}]$, and [Ca II]. We report on a study of nebular lines during the current ongoing minimum of R CrB that started in 2007 July.

\section{Present observations: $2007-2011$ minimum of $\mathrm{R} \mathbf{C r}$}

Spectroscopic observations have been obtained at various resolutions using HFOSC 2m Chandra telescope at Hanle, Ladhak $(\mathrm{R}=2,300)$, 2.7m Harlan Smith telescope at McDonald Observatory $(\mathrm{R}=30-60,000)$ and 10-m Keck1 telescope $(\mathrm{R}=30,000)$. Forbidden lines seen are: $[\mathrm{O} \mathrm{II}],[\mathrm{N} \mathrm{II}],[\mathrm{Ca} \mathrm{II}]$, and [S II]. They show double peaks at high resolution and are separated by $170 \mathrm{~km} / \mathrm{s}$. The FWHM of each peak is $140 \mathrm{~km} / \mathrm{s}$.

Electron density and temperature (nebular lines): [S II] $\lambda 6717$ and $\lambda 6731$ are present in the HCT spectra obtained in 2009 Feb 12 (Fig.1). The 6717/6731 flux ratio of 1.4 suggests $N_{e}$ of about $50-100 \mathrm{~cm}^{-3}$. The Keck high-resolution spectrum obtained on 2010 June 20 covers the region of the [O II] lines (Figure 2). Since the lines are double peaked, the red and blue components of the [O II] $\lambda 3729$ and $\lambda 3726$ lines, respectively, are blended. To estimate their flux ratio, we assume that [O II] profiles and the flux ratio of the blue and red components are similar to that of the [N II] $\lambda 6583$ line. We arrived at the $[\mathrm{O} \mathrm{II}] \mathrm{F}(3729) / \mathrm{F}(3726)$ flux ratio of 1.21 by scaling the [N II] $\lambda 6583$ line to match the $[\mathrm{O} \mathrm{II}]$ profiles that suggested $N e$ of $100 \mathrm{~cm}^{-3}$, indicating a low density nebula. The [N II] $\lambda 6548 \& 6583$ are very close to the expected ratio $(1: 3)$ but the $[\mathrm{N} \mathrm{II}] \lambda 5755$ line 

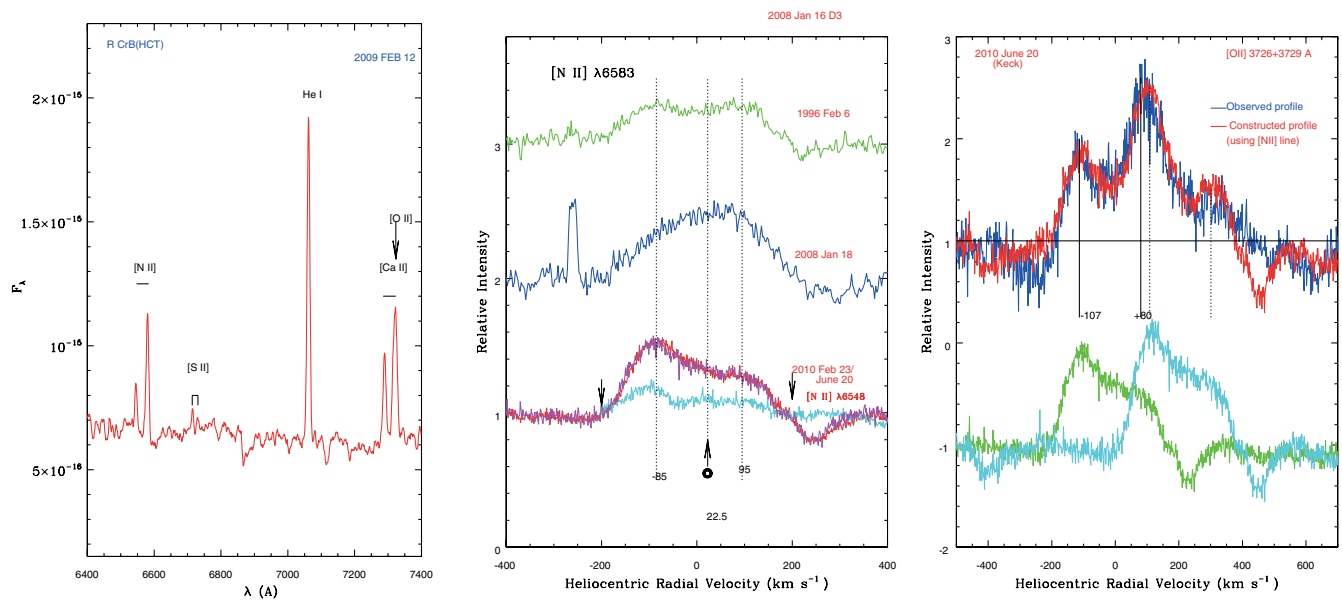

Figure 1. Left panel: Low resolution spectra showing [S II], [N II] lines on 2009 Feb 12. Middle panel: Keck spectrum obtained at deep minimum on 2010 Feb 23 showing [N II] lines (red \& cyan). The two peaks in [N II] profiles are obvious. The [N II] line region was amplified, and shifted up, showing variability in profile (and flux). The radial velocity range of [N II]. The arrow at $22 \mathrm{~km} / \mathrm{s}$ denotes the normal stellar photospheric radial velocity. Right panel: the [O II] blend of 3726,3729 and the process of estimating the line ratio using the [N II] $\lambda 6583$ line is illustrated.

was never seen. By using an upper limit to the flux of the latter line, Te $<7500 \mathrm{~K}$ is obtained. The nebular extent is estimated to be about $2308 \mathrm{R}_{\text {star }}\left(0.59^{\prime \prime}\right)$ - by adopting a distance of $1.6 \mathrm{kpc}$ which is much bigger than the location of the hot dust $(\sim 600 \mathrm{~K})$. Once ionized, at these low density, the nebula remains ionized, even if the source of ionization is cut off, (for about $10^{4}$ years).

Variability of $[\mathrm{N} \mathrm{II}]$ during the minimum: the $[\mathrm{N} \mathrm{II}] \lambda 6583$ flux seemed to have varied along with the profile during the current minimum. The flux in 1995-96 minimum was $1.96 \times 10^{-17} \mathrm{~W} \mathrm{~m}^{-2}$. The same flux is also seen in the initial part of the current minimum but has decreased in a two years period from $1.97 \times 10^{-17}$ to $1.16 \times 10^{-17} \mathrm{~W} \mathrm{~m}^{-2}$. The red peak flux decreased by 2010 June 20 . The velocity range on the red side also decreased from 200 to $170 \mathrm{~km} / \mathrm{s}$. What caused the eating of the red side of the profile? (This is seen in other permitted line profiles too - (Na I D, Ca II, He I, etc.) - movement of a dust cloud? suggests that the blue emission (expanding gas) is closer to us than new dust?

\section{Discussion \& Questions}

Forbidden lines (low density gas) are general features of all RCBs - many unanswered questions remain. Why does low-density $\left(N_{e} \sim 100 \mathrm{~cm}^{-3}\right)$ nebular gas has such high velocities of expansion (rotation)?. If it is ejected like a $\mathrm{PN}$, it should have a much lower expansion velocity of $\sim 20-30 \mathrm{~km} / \mathrm{s}$ (or even born-again AGB?). Could it have been ejected in the course of white dwarf mergers? - some merger models of CO $\left(0.8 \mathrm{M}_{\odot}\right)+$ He $\left(0.4 \mathrm{M}_{\odot}\right)$ white dwarfs, show large mass loss and formation of hot corona and a disk around the resultant compact object.

\section{Acknowledgements}

N. Kameswara Rao would like to thank Anibal García-Hernández for help with the poster preparation. We also would like to thank Philip McQueen, Anita Cochran, and D. K. Sahu for getting the observations for us. 